\title{
Fourth Order Runge-Kutta Method Based On Geometric Mean for Hybrid Fuzzy Initial Value Problems
}

\author{
R. Gethsi Sharmila \\ Assistant Professor Department of Mathematics Bishop Heber College (Autonomous), Tiruchirappalli -620 017, \\ Tamilnadu, India.
}

\begin{abstract}
In this paper, a numerical algorithm for solving hybrid fuzzy initial value problem using the Fourth Order Runge-Kutta method based on Geometric Mean (RK4GM) is proposed. The algorithm is illustrated by solving hybrid fuzzy initial value problems using triangular fuzzy number. It is compared with the classical fourth order Runge - Kutta method. The results show that the proposed fourth order Runge-Kutta method based on Geometric Mean works well for solving hybrid fuzzy initial value problems.
\end{abstract}

Keywords: Numerical solution, Hybrid Fuzzy Initial Value Problems, Triangular fuzzy number, Fourth order Runge-Kutta method, Geometric Mean

\section{Introduction}

Fuzzy set theory is a tool that makes possible to describe vague and uncertain notions. Fuzzy Differential Equation (FDE) models have wide range of applications in many branches of engineering and in the field of medicine. The concept of a fuzzy derivative was first introduced by Chang and Zadeh [5], later Dubois and Prade [6] defined the fuzzy derivative by using Zadeh's extension principle and then followed by Puri and Ralescu [23]. Fuzzy differential equations have been suggested as a way of modelling uncertain and incompletely specified systems and were studied by many researchers $[9,10]$. The existence of solutions of fuzzy differential equations has been studied by several authors [3, 4]. It is difficult to obtain an exact solution for fuzzy differential equations and hence several numerical methods where proposed $[11,12,14,18]$. Abbasbandy and Allahviranloo [2] developed numerical algorithms for solving fuzzy differential equations based on Seikkala's derivative of fuzzy process [25]. Runge-Kutta method for fuzzy differential equation has been studied by many authors $[1,19]$. The numerical solution to hybrid fuzzy systems has been studied by the authors [8, 20,21,22 and 24].

Abdul-Majid Wazwaz [3] introduced a comparison of modified Runge-Kutta formulas based on a variety of means. Evans and Yaacub [7] compared the numerical o.d.e. solvers based on arithmetic and geometric means for first order IVPs. Murugesan et al. [16] compared fourth order RK methods based on variety of means and concluded that RK4CeM works very well to solve system of IVPs and they also developed [17] a new embedded RK method based on AM and CeM. The applicability of the RK4CeM : Division by zero, Error in RK4CeM formulae, and Stability analysis are discussed by Murugesan et al. [15].

Hybrid systems are devoted to modeling, design, and validation of interactive systems that are capable of controlling complex systems which have discrete event dynamics as well as continuous time dynamics can be modeled by hybrid systems. The differential systems containing fuzzy valued functions and interaction with a discrete time controller are named hybrid fuzzy differential systems. The numerical solution to hybrid fuzzy systems has been studied by the authors [8, 20, 21, 22 and 24].

In this paper, the RK4GM is applied to solve hybrid fuzzy initial value problems.

The structure of the paper is organized as follows: In Section 2, some necessary notations and definitions of fuzzy set theory, hybrid fuzzy differential equations, the fourth order Runge-Kutta formula based on Geometric Mean to solve IVPs are given. In Section 3 Hybrid Fuzzy Initial Value Problem is defined and the numerical algorithm for solving the fuzzy initial value problems by the fourth order Runge-Kutta method based on Geometric Mean are given. Section 4 contains the numerical example. The conclusion is given in section 5 .

\section{Preliminaries}

In this section, some of the basic definitions of fuzzy numbers and Runge-Kutta methods are reviewed.

\subsection{Definitions and notations}

Denote by $E^{1}$ the set of all functions $\mathrm{u}: \mathrm{R} \rightarrow[0,1]$ such that (i) u is normal, that is, there exist an $x_{0} \in R$ such that $u\left(x_{0}\right)=1$, (ii) $\mathrm{u}$ is a fuzzy convex, that is, for $x, y \in R$ and $0 \leq \lambda \leq 1$, 
$\mathrm{u}(\lambda x+(1-\lambda) y) \geq \min \{u(x), u(y)\}$,(iii) $\mathrm{u}$ is upper semi-continuous, and (iv) $[u]^{0} \equiv$ the closure of $\{x \in R: u(x)>0\}$ is compact. For $0<r \leq 1$, we define $[u]_{r}=\{x \in R: u(x) \geq r\}$. An example of a $u \in E^{1}$ is given by

$$
u(x)= \begin{cases}4 x-3, & \text { if } x \in(0.75,1], \\ -2 x+3, & \text { if } x \in(1,1.5], \\ 0, & \text { if } x \notin(0.75,1.5],\end{cases}
$$

The $\mathrm{r}$ - level sets of $\mathrm{u}$ in (2.1) are given by

$$
[u]_{r}=[0.75+0.25 r, 1.5-0.5 r]
$$

For later purpose, we define $\hat{0} \in E^{1}$ as $\hat{O}(x)=1$, if $\mathrm{x}=0$ and $\hat{O}(x)=0$, if $\mathrm{x} \neq 0$. Next we review the seikkala derivative [25] of $x: I \rightarrow E^{1}$ where $I \subset R$ is an interval. If $[x(t)]_{r}=[\underline{x}(t ; r), \bar{x}(t ; r)]$ for all $t \in I$ and $r \in[0,1]$, then $\left[x^{\prime}(t)\right]_{r}=\left[\underline{x}(t ; r), \bar{x}^{\prime}(t ; r)\right]$ if $x^{\prime}(t) \in E^{1}$. Next consider the initial value problem (IVP)

$$
u(y)=\left\{\begin{array}{l}
y^{\prime}(t)=f(t, y(t)), \\
y(0)=y,
\end{array}\right.
$$

where $\mathrm{f}:[0, \infty) \times \mathrm{R} \rightarrow \mathrm{R}$ is continuous. We would like to interpret (2.3) using the Seikkala derivative and $x_{0}$ $\in E^{1}$. Let $\left[x_{0}\right]_{r}=\left[\underline{x}_{0}(r), \bar{x}_{0}(r)\right]$ and $[x(t)]_{r}=[\underline{x}(t ; r), \bar{x}(t ; r)]$ by the zadeh extension principle we get $f:[0, \infty) \times E^{1} \rightarrow E^{1}$ Where

$[f(t, y)]_{r}=[\min \{f(t, u): u \in[\underline{x}(t ; r), \bar{x}(t ; r)]\}, \max \{f(t, u): u \in[\underline{x}(t ; r), \bar{x}(t ; r)]\}]$.

Then $x:[0, \infty) \rightarrow E^{1}$ is a solution of (2.3) using the seikkala derivative and $x_{0} \in E^{1}$ if

$$
\begin{aligned}
& \underline{x}^{\prime}(t ; r)=\min \{f(t, u): u \in[\underline{x}(t ; r), \bar{x}(t ; r)]\}, \underline{x}(0 ; r)=\underline{x}_{0}(r) \\
& \bar{x}^{\prime}(t ; r)=\max \{f(t, u): u \in[\underline{x}(t ; r), \bar{x}(t ; r)]\}, \bar{x}(0 ; r)=\bar{x}_{0}(r)
\end{aligned}
$$

For all $t \in[0, \infty)$ and $r \in[0,1]$.

Consider a function $f:[0, \infty) \times R \times R \rightarrow R$ which is continuous and the IVP

$$
\left\{\begin{array}{l}
y^{\prime}(t)=f(t, y(t), k), \\
y(0)=y_{0}
\end{array}\right.
$$

to interpret(2.4) using the seikkala derivative and $y_{0}, k \in E^{1}$, by the zadeh extention principle we use $f:[0, \infty) \times E^{1} \times E^{1} \rightarrow E^{1}$ where

$$
\begin{aligned}
{[f(t, y, k)]_{r}=} & {\left[\min \left\{f\left(t, u, u_{k}\right): u \in[\underline{y}(t ; r), \bar{y}(t ; r)], u_{k} \in[\underline{k}(r), \bar{k}(r)]\right\},\right.} \\
& \max \left\{f\left(t, u, u_{k}\right): u \in[\underline{y}(t ; r), \bar{y}(t ; r)], u_{k} \in[\underline{k}(r), \bar{k}(r)]\right\},
\end{aligned}
$$

where $k_{r}=[\underline{k}(r), \bar{k}(r)]$ then $y:[0, \infty) \rightarrow E^{1}$ is a solution of (2.4) using the seikkala derivative and $y_{0} k \in E^{1}$ if

$\underline{y^{\prime}}(t, y ; r)=\min \left\{f\left(t, u, u_{k}\right) \backslash u \in[\underline{y}(t ; r), \bar{y}(t ; r)], u_{k} \in[\underline{k}(r), \bar{k}(r)]\right\}, \underline{\mathrm{y}}(0 ; r)=\underline{y}_{0}(r)$

$\overline{y^{\prime}}(t, y ; r)=\max \left\{f\left(t, u, u_{k}\right) \backslash u \in[\underline{y}(t ; r), \bar{y}(t ; r)], u_{k} \in[\underline{k}(r), \bar{k}(r)]\right\}, \overline{\mathrm{y}}(0 ; r)=\overline{y_{0}}(r)$

for all $\mathrm{t} \in[0, \infty)$ and $r \in[0,1]$. 


\subsection{Runge - Kutta Method for Initial Value Problem}

Consider the initial value problem

$$
\begin{aligned}
& y^{\prime}(t)=f(t, y(t)) ; \mathrm{a} \leq \mathrm{t} \leq \mathrm{b} \\
& \mathrm{y}(\mathrm{a})=r,
\end{aligned}
$$

The basis of all Runge-Kutta method is to express the difference between the value of y at $t_{n+1}$ and $t_{n}$ as

$$
y_{n+1}-y_{n}=\sum_{i=1}^{m} w_{i} k_{i}
$$

where for $\mathrm{i}=1,2, \ldots, \mathrm{m}, \mathrm{w}_{\mathrm{i}}$ 's are constants and $k_{i}=h f\left(t_{n}+c_{i} h, y_{n}+\sum_{j=1}^{i-1} a_{i j} k_{j}\right)$

Equations (2.6) is to be exact for powers of h through $h^{m}$, because it is to be coincident with Taylor series of order $\mathrm{m}$.

\subsection{The Fourth Order Runge-Kutta method Based On Geometric Mean For Solving Initial Value Problem}

Evans [7] developed a new fourth order RK method for solving IVPs is derived by replacing the arithmetic means in the formula $\mathrm{y}_{\mathrm{n}+1}=\mathrm{y}_{\mathrm{n}}+\frac{\mathrm{h}}{6}\left(\mathrm{k}_{1}+2 \mathrm{k}_{2}+2 \mathrm{k}_{3}+\mathrm{k}_{4}\right)$ where

$$
\mathrm{k}_{\mathrm{i}}=\mathrm{f}\left(\mathrm{y}_{\mathrm{n}}+\mathrm{h} \sum_{\mathrm{j}=1}^{\mathrm{i}-1} \mathrm{a}_{\mathrm{ij}} \mathrm{k}_{\mathrm{j}}\right)
$$

by their geometric means i.e. $\left(\mathrm{k}_{1}+\mathrm{k}_{2}\right) / 2 \approx \sqrt{\mathrm{k}_{1} \mathrm{k}_{2}}$ etc. to yield initially a low order accuracy formula. For $y^{\prime}=f(t, y)$, the fourth order Runge-Kutta methods using Geometric Mean can be written in the form

$$
y_{n+1}=y_{n}+\frac{h}{3}\left[\sum_{i=1}^{3} \text { Means }\right]
$$

where means includes Geometric Mean(GM) which involves $k_{i}, 1 \leq i \leq 4$, where,

$k_{1}=f\left(t_{n}, y_{n}\right), k_{2}=f\left(t_{n}+a_{1} h, y_{n}+a_{1} h k_{1}\right), k_{3}=f\left(t_{n}+\left(a_{2}+a_{3}\right) h, y_{n}+a_{2} h k_{1}+a_{3} h k_{2}\right)$

$k_{4}=f\left(t_{n}+\left(a_{4}+a_{5}+a_{6}\right) h, y_{n}+\left(a_{4} h k_{1}+a_{5} h k_{2}+a_{6} h k_{3}\right)\right)$

(2.8)

where the parameters for

Geometric Mean : $a_{1}=\frac{1}{2}, a_{2}=\frac{-1}{16}, a_{3}=\frac{9}{16}, a_{4}=\frac{-1}{8}, a_{5}=\frac{5}{24}, a_{6}=\frac{11}{12}$.

The fourth order formulae based on Runge-Kutta scheme using Geometric Mean is as follows:

Geometric Mean : $y_{n+1}=y_{n}+\frac{h}{3}\left(\sqrt{\left|k_{1} k_{2}\right|}+\sqrt{\left|k_{2} k_{3}\right|}+\sqrt{\left|k_{3} k_{4}\right|}\right)$

With the grid points

$a=t_{0}<t_{1}<\ldots<t_{N}=b$ and $h=\frac{(b-a)}{N}=t_{i+1}-t_{i}$

The LTE for this formula is given as follows:

$$
\operatorname{LTE}_{\mathrm{GM}}=\frac{\mathrm{h}^{5}}{184320}\left[-64 \mathrm{f}^{4} \mathrm{f}_{\mathrm{yyyy}}-208 \mathrm{f}^{3} f_{y} f_{y y y}-3744 \mathrm{f}^{3} \mathrm{f}_{\mathrm{yy}}^{2}-1974 \mathrm{f}^{2} \mathrm{f}_{\mathrm{y}}^{2} \mathrm{f}_{\mathrm{yy}}+3561 \mathrm{ff}_{\mathrm{y}}^{4}\right]+0\left(\mathrm{~h}^{6}\right)
$$

Theorem 2.1. Let $f(t, y)$ belongs to $\mathrm{C}^{4}[\mathrm{a}, \mathrm{b}]$ and let it's partial derivatives are bounded and assume that there exists L, M, positive constants such that

$$
|f(t, y)|<M, \quad\left|\frac{\partial^{i+j} f}{\partial t^{i} \partial y^{j}}\right|<\frac{L^{i+j}}{M^{j-1}}, i+j<m
$$


then in terms of the error bound due to Lotkin (see Lambert [13], we have a strict upper bound (with respect to y only) as in the fourth order Runge-Kutta method based on

Geometric Mean $\quad:\left|y\left(t_{i+1}\right)-y_{i+1}\right|=\left|L T E_{G M}\right| \leq \frac{2429}{184320} h^{5} M L^{4}+O\left(h^{6}\right)$

Theorem 2.2 Let f satisfy

$$
|f(t, v)-f(t, \bar{v})| \leq g(t,|v-\bar{v}|), t \geq 0, v, \bar{v} \in R .
$$

where $g: R_{+} \times R_{+} \rightarrow R_{+}$is a continuous mapping such that $r \rightarrow g(t, r)$ is non-decreasing and the initial value problem

$$
u^{\prime}(t)=g(t, u(t)), u(0)=u_{0} .
$$

has a solution on $\mathrm{R}_{+}$for $\mathrm{u}_{0}>0$ and that $\mathrm{u}(\mathrm{t})=0$ is the only solution of (3.4) for $\mathrm{u}_{0}=0$. Then the fuzzy initial value problem (2.3) has a unique fuzzy solution. Refer [25].

Consider the hybrid fuzzy initial value problem

\section{The Hybrid Fuzzy Initial Value Problem}

$$
\left\{\begin{array}{l}
y^{\prime}(t)=f\left(t, y(t), \lambda_{k}\left(y_{k}\right)\right), t \in\left[t_{k}, t_{k+1}\right], \\
y\left(t_{k}\right)=y_{k},
\end{array}\right.
$$

where ' denotes seikkala differentiation

$0 \leq t_{0}<t_{1}<\ldots .<t_{k}<\ldots t_{k} \rightarrow \infty, f \in C\left[R^{+} \times E^{1} \times E^{1}, E^{1}\right], \lambda_{k} \in C\left[E^{1}, E^{1}\right]$

To be specific the system look like

$$
y^{\prime}(t)=\left\{\begin{array}{l}
y_{0}^{\prime}(t)=f\left(t, y_{0}(t), \lambda_{0}\left(y_{0}\right)\right), y_{0}\left(t_{0}\right)=y_{0}, \quad t_{0} \leq t \leq t_{1}, \\
y_{1}^{\prime}(t)=f\left(t, y_{1}(t), \lambda_{1}\left(y_{1}\right)\right), y_{1}\left(t_{1}\right)=y_{1}, \quad t_{1} \leq t \leq t_{2}, \\
\cdots \\
y_{k}^{\prime}(t)=f\left(t, y_{k}(t), \lambda_{k}\left(y_{k}\right)\right), y_{k}\left(t_{k}\right)=y_{k}, \quad t_{k} \leq t \leq t_{k+1}, \\
\cdots
\end{array}\right.
$$

Assuming that the existence and uniqueness of solution of (3.1) hold for each $\left[t_{k}, t_{k+1}\right]$, by the solution of (3.1) we mean the following function:

$$
y(t)=y\left(t, t_{0}, y_{0}\right)=\left\{\begin{array}{l}
y_{0}(t), t_{0} \leq t \leq t_{1}, \\
y_{1}(t), t_{1} \leq t \leq t_{2}, \\
\cdots \\
y_{k}(t), t_{k} \leq t \leq t_{k+1}, \\
\cdots
\end{array}\right.
$$

We note that the solution of (3.1) are piecewise differentiable in each in each interval for $t \in\left[t_{k}, t_{k+1}\right]$ for fixed $x_{k} \in E^{1}, k=0,1,2, \ldots$

Therefore we may replace (3.1) by an equivalent system

$$
\underline{y^{\prime}}(t)=\underline{f}\left(t, \mathrm{y}, \lambda_{k}\left(y_{k}\right) \equiv F_{k}[t, \underline{y}, \bar{y}], \underline{y}\left(t_{k}\right) \in \underline{y_{k}}, \overline{y^{\prime}}(t)=\bar{f}\left(t, \mathrm{y}, \lambda_{k}\left(y_{k}\right) \equiv G_{k}[t, \underline{y}, \bar{y}], \bar{y}\left(t_{k}\right) \in \overline{y_{k}},\right.\right.
$$

which possesses a unique solution $(y, \bar{y})$ which is a fuzzy function. That is for each $\mathrm{t}$, the pair $(\underline{y}(t ; r), \bar{y}(t ; r))$ is a fuzzy number, where, $\underline{y}(t ; r), \bar{y}(t ; r)$ are respectively the solutions of the parametric form given by 


$$
\begin{aligned}
& \underline{y^{\prime}}(t ; r)=F_{k}[t, \underline{y}(t ; r), \bar{y}(t ; r)], \underline{y}\left(t_{k} ; r\right) \in \underline{\underline{y_{k}}}(r), \\
& \overline{y^{\prime}}(t ; r)=G_{k}[t, \underline{y}(t ; r), \bar{y}(t ; r)], \bar{y}\left(t_{k} ; r\right) \in \overline{\overline{y_{k}}}(r), \text { for } r \in[0,1]
\end{aligned}
$$

\subsection{The Fourth Order Runge-Kutta method based on Geometric Mean For Hybrid Fuzzy Initial Value \\ Problem}

In this section, for a hybrid fuzzy initial value problem (3.1) the fourth order Runge - Kutta method based on Geometric Mean is developed. Here the assumption is that the exixtence and uniqueness of the solutions of (3.1) hold for each $\left[t_{k}, t_{k+1}\right]$.

For a fixed $\mathrm{r}$, to integrate the system (3.3) in $\left[t_{0}, t_{1}\right],\left[t_{1}, t_{2}\right], \ldots,\left[t_{k}, t_{k+1}\right], \ldots$, we replace each interval by a set of $N_{k}+1$ discrete equally spaced grid points at which the exact solution $Y(t ; r)=(\underline{Y}(t ; r), \bar{Y}(t ; r))$ is approximated by some $\left(\underline{y}_{k}(t ; r), \bar{y}_{k}(t ; r)\right)$

For each chosen grid points on $\left[t_{k}, t_{k+1}\right]$ at $t_{k, n}=t_{k}+n h_{k}, \mathrm{~h}_{k}=\frac{t_{k+1}-t_{k}}{N_{k}}, 0 \leq \mathrm{n} \leq \mathrm{N}_{k}$.

Let $\left[\underline{Y}_{k}(t ; r), \bar{Y}_{k}(t ; r)\right] \equiv(\underline{y}(t ; r), \bar{y}(t ; r)),\left(\underline{Y}_{k}(t ; r), \bar{Y}_{k}(t ; r)\right)$ and $\left(\underline{y}_{k}(t ; r), \bar{y}_{k}(t ; r)\right)$ may be denoted respectively by $\left(\underline{Y}_{k, n}(r), \bar{Y}_{k, n}(r)\right)$ and $\left(\underline{y}_{k, n}(r), \bar{y}_{k, n}(r)\right)$. We allow the $N_{k}$ 's to vary over the $\left[t_{k}, t_{k+1}\right]$ 's so that the $h_{k}$ 's may be comparable.

The fourth order Runge - Kutta method based on Geometric mean is a an approximation of $\underline{Y}_{k}^{\prime}(t ; \mathrm{r})$ and $\bar{Y}_{k}^{\prime}(t ; \mathrm{r})$. To develop the fourth order Runge - Kutta method based on Geometric Mean for (2.3), define $\underline{k}_{1}\left(t_{k, n} ; y_{k, n}(r)\right)=\min \left\{f\left(t_{k, n}, u, \lambda_{k}\left(u_{k}\right)\right) \backslash u \in\left[\underline{y}_{k, n}(r), \bar{y}_{k, n}(r)\right], \mathrm{u}_{k} \in\left[\underline{y}_{k, 0}(r), \bar{y}_{k, 0}(r)\right]\right\}$ $\bar{k}_{1}\left(t_{k, n} ; y_{k, n}(r)\right)=\max \left\{f\left(t_{k, n}, u, \lambda_{k}\left(u_{k}\right)\right) / u \in\left[\underline{y}_{k, n}(r), \bar{y}_{k, n}(r)\right], \mathrm{u}_{k} \in\left[\underline{y}_{k, 0}(r), \bar{y}_{k, 0}(r)\right]\right\}$ $\underline{k}_{2}\left(t_{k, n} ; y_{k, n}(r)\right)=\min \left\{f\left(t_{k, n}+\frac{h_{k}}{2}, u, \lambda_{k}\left(u_{k}\right)\right) \backslash u \in\left[\underline{z}_{k 1}\left(t_{k, n} ; y_{k, n}(r)\right), \bar{z}_{k 1}\left(t_{k, n} ; y_{k, n}(r)\right)\right], \mathrm{u}_{k} \in\left[\underline{y}_{k, 0}(r), \bar{y}_{k, 0}(r)\right]\right\}$ $\underline{k}_{3}\left(t_{k, n} ; y_{k, n}(r)\right)=\min \left\{f\left(t_{k, n}+\frac{h_{k}}{2}, u, \lambda_{k}\left(u_{k}\right)\right) \backslash u \in\left[\underline{z}_{k 2}\left(t_{k, n} ; y_{k, n}(r)\right), \bar{z}_{k 2}\left(t_{k, n} ; y_{k, n}(r)\right)\right], \mathrm{u}_{k} \in\left[\underline{y}_{k, 0}(r), \bar{y}_{k, 0}(r)\right]\right\}$ $\bar{k}_{3}\left(t_{k, n} ; y_{k, n}(r)\right)=\max \left\{f\left(t_{k, n}+\frac{h_{k}}{2}, u, \lambda_{k}\left(u_{k}\right)\right) \backslash u \in\left[\underline{z}_{k 2}\left(t_{k, n} ; y_{k, n}(r)\right), \bar{z}_{k 2}\left(t_{k, n} ; y_{k, n}(r)\right)\right], \mathrm{u}_{k} \in\left[\underline{y}_{k, 0}(r), \bar{y}_{k, 0}(r)\right]\right\}$ $\bar{k}_{2}\left(t_{k, n} ; y_{k, n}(r)\right)=\max \left\{f\left(t_{k, n}+\frac{h_{k}}{2}, u, \lambda_{k}\left(u_{k}\right)\right) \backslash u \in\left[\underline{z}_{k 1}\left(t_{k, n} ; y_{k, n}(r)\right), \bar{z}_{k 1}\left(t_{k, n} ; y_{k, n}(r)\right)\right], \mathrm{u}_{k} \in\left[\underline{y}_{k, 0}(r), \bar{y}_{k, 0}(r)\right]\right\}$ $\underline{k}_{4}\left(t_{k, n} ; y_{k, n}(r)\right)=\min \left\{f\left(t_{k, n}+h_{k}, u, \lambda_{k}\left(u_{k}\right)\right) \backslash u \in\left[\underline{z}_{k 3}\left(t_{k, n} ; y_{k, n}(r)\right), \bar{z}_{k 3}\left(t_{k, n} ; y_{k, n}(r)\right)\right], \mathrm{u}_{k} \in\left[\underline{y}_{k, 0}(r), \bar{y}_{k, 0}(r)\right]\right\}$ $\bar{k}_{4}\left(t_{k, n} ; y_{k, n}(r)\right)=\max \left\{f\left(t_{k, n}+h_{k}, u, \lambda_{k}\left(u_{k}\right)\right) \backslash u \in\left[\underline{z}_{k 3}\left(t_{k, n} ; y_{k, n}(r)\right), \bar{z}_{k 3}\left(t_{k, n} ; y_{k, n}(r)\right)\right], \mathrm{u}_{k} \in\left[\underline{y}_{k, 0}(r), \bar{y}_{k, 0}(r)\right]\right\}$ where

$$
\begin{aligned}
& \underline{z}_{k 1}\left(t_{k, n} ; y_{k, n}(r)\right)=\underline{y}_{k, n}(r)+\frac{1}{2} h_{k} \underline{k}_{1}\left(t_{k, n} ; y_{k, n}(r)\right), \bar{z}_{k 1}\left(t_{k, n} ; y_{k, n}(r)\right)=\bar{y}_{k, n}(r)+\frac{1}{2} h_{k} \bar{k}_{1}\left(t_{k, n} ; y_{k, n}(r)\right) \\
& \underline{z}_{k 2}\left(t_{k, n} ; y_{k, n}(r)\right)=\underline{y}_{k, n}(r)+\frac{-1}{16} h_{k} \underline{k}_{1}\left(t_{k, n} ; y_{k, n}(r)\right)+\frac{9}{16} h_{k} \underline{k}_{2}\left(t_{k, n} ; y_{k, n}(r)\right) \\
& \bar{z}_{k 2}\left(t_{k, n} ; y_{k, n}(r)\right)=\bar{y}_{k, n}(r)+\frac{-1}{16} h_{k} \bar{k}_{1}\left(t_{k, n} ; y_{k, n}(r)\right)+\frac{9}{16} h_{k} \bar{k}_{2}\left(t_{k, n} ; y_{k, n}(r)\right) \\
& \underline{z}_{k 3}\left(t_{k, n} ; y_{k, n}(r)\right)=\underline{y}_{k, n}(r)+\frac{-1}{8} h_{k} \underline{k}_{1}\left(t_{k, n} ; y_{k, n}(r)\right)+\frac{5}{24} h_{k} \underline{k}_{2}\left(t_{k, n} ; y_{k, n}(r)\right)+\frac{11}{12} h_{k} \underline{k}_{3}\left(t_{k, n} ; y_{k, n}(r)\right) \\
& \bar{z}_{k 3}\left(t_{k, n} ; y_{k, n}(r)\right)=\bar{y}_{k, n}(r)+\frac{-1}{8} h_{k} \bar{k}_{1}\left(t_{k, n} ; y_{k, n}(r)\right)+\frac{5}{24} h_{k} \bar{k}_{2}\left(t_{k, n} ; y_{k, n}(r)\right)+\frac{11}{12} h_{k} \bar{k}_{3}\left(t_{k, n} ; y_{k, n}(r)\right)
\end{aligned}
$$

Next define 


$$
\begin{aligned}
S_{k}\left[t_{k, n} ; \underline{y}_{k, n}(r), \bar{y}_{k, n}(r)\right]= & \sqrt{\left|\underline{k}_{1}\left(t_{k, n} ; y_{k, n}(r)\right) \underline{k}_{2}\left(t_{k, n} ; y_{k, n}(r)\right)\right|}+\sqrt{\left|\underline{k}_{2}\left(t_{k, n} ; y_{k, n}(r)\right) \underline{k}_{3}\left(t_{k, n} ; y_{k, n}(r)\right)\right|} \\
& +\sqrt{\left|\underline{k}_{3}\left(t_{k, n} ; y_{k, n}(r)\right) \underline{k}_{4}\left(t_{k, n} ; y_{k, n}(r)\right)\right|} \\
T_{k}\left[t_{k, n} ; \underline{y}_{k, n}(r), \bar{y}_{k, n}(r)\right]= & \sqrt{\left|\bar{k}_{1}\left(t_{k, n} ; y_{k, n}(r)\right) \bar{k}_{2}\left(t_{k, n} ; y_{k, n}(r)\right)\right|}+\sqrt{\left|\bar{k}_{2}\left(t_{k, n} ; y_{k, n}(r)\right) \bar{k}_{3}\left(t_{k, n} ; y_{k, n}(r)\right)\right|} \\
& +\sqrt{\bar{k}_{3}\left(t_{k, n} ; y_{k, n}(r)\right) \bar{k}_{4}\left(t_{k, n} ; y_{k, n}(r)\right) \mid}
\end{aligned}
$$

The exact solutions at $\mathrm{t}_{\mathrm{k}, \mathrm{n}+1}$ is given by

$$
\underline{Y}_{k, n+1}(r) \approx \underline{\mathrm{Y}}_{k, n}(\mathrm{r})+\frac{1}{3} h_{k} S_{k}\left[t_{k, n} ; \underline{Y}_{k, n}(r), \bar{Y}_{k, n}(r)\right], \bar{Y}_{k, n+1}(r) \approx \overline{\mathrm{Y}}_{k, n}(\mathrm{r})+\frac{1}{3} h_{k} T_{k}\left[t_{k, n} ; \underline{Y}_{k, n}(r), \bar{Y}_{k, n}(r)\right]
$$

The approximate solutions at $\mathrm{t}_{\mathrm{k}, \mathrm{n}+1}$ is given by

$$
\underline{y}_{k, n+1}(r) \approx \underline{y}_{k, n}(\mathrm{r})+\frac{1}{3} h_{k} S_{k}\left[t_{k, n} ; \underline{y}_{k, n}(r), \bar{y}_{k, n}(r)\right], \bar{y}_{k, n+1}(r) \approx \bar{y}_{k, n}(\mathrm{r})+\frac{1}{3} h_{k} T_{k}\left[t_{k, n} ; \underline{y}_{k, n}(r), \bar{y}_{k, n}(r)\right]
$$

Theorem 3.1. Consider the systems (3.2) and (3.5). For a fixed $\mathrm{k} \in \mathrm{Z}^{+}$and $\mathrm{r} \in[0,1]$,

$$
\lim _{h_{0}, \ldots, h_{k} \rightarrow 0} \underline{y}_{k, N_{k}}(r)=\underline{x}\left(t_{k+1} ; r\right), \lim _{h_{0}, \ldots, h_{k} \rightarrow 0} \bar{y}_{k, N_{k}}(r)=\bar{x}\left(t_{k+1} ; r\right),
$$

\section{Numerical Example}

Before illustrating the numerical solution of Hybrid Fuzzy Initial Value Problem (HFIVP), first we recall the fuzzy IVP;

$$
\left\{\begin{array}{l}
y^{\prime}(t)=y(t), t \in[0,1] \\
y(0 ; r)=[0.75+0.25 r, 1.125-0.125 r], 0 \leq \mathrm{r} \leq 1
\end{array}\right.
$$

The exact solution of (4.1) is given by

At $\mathrm{t}=1$ we get

$$
Y(t ; r)=\left[(0.75+0.25 r) e^{t},(1.125-0.125 r) e^{t}\right], 0 \leq \mathrm{r} \leq 1 \quad 0 \leq \mathrm{r} \leq 1 .
$$

$$
Y(1 ; r)=\left[(0.75+0.25 r) e^{1},(1.125-0.125 r) e^{1}\right], 0 \leq \mathrm{r} \leq 1
$$

By the fourth order Runge -Kutta method based on Geometric Mean with $\mathrm{N}=2$, (4.1) gives the approximate solution as follows:

$$
\begin{gathered}
y(1.0 ; r)=\left[(0.75+0.25 r)\left(c_{0,1}\right)^{2},(1.125-0.125 r)\left(c_{0,1}\right)^{2}\right], 0 \leq \mathrm{r} \leq 1 \\
\text { where } c_{0,1}=1+\frac{h}{3}\left[\begin{array}{l}
\sqrt{1+\frac{1}{2} h}+\sqrt{1+h+\frac{17}{32} h^{2}+\frac{9}{64} h^{3}} \\
+\sqrt{1+\frac{3}{2} h+\frac{43}{32} h^{2}+\frac{105}{128} h^{3}+\frac{147}{512} h^{4}+\frac{297}{4096} h^{5}}
\end{array}\right]
\end{gathered}
$$

Example 4.1. Next consider the following hybrid fuzzy initial value problem,

$$
\left\{\begin{array}{l}
y^{\prime}(t)=y(t)+m(t) \lambda_{k}\left(y\left(t_{k}\right)\right), t \in\left[t_{k}, t_{k+1}\right], t_{k}=k, k=0,1,2,3, \ldots, \\
y(t ; r)=\left[(0.75+0.25 r) e^{t},(1.125-0.125 r) e^{t}\right], 0 \leq r \leq 1
\end{array}\right.
$$

where

$$
m(t)= \begin{cases}2(t(\bmod 1)) & \text { if } t(\bmod 1) \leq 0.5, \\ 2(1-t(\bmod 1)) & \text { if } t(\bmod 1) \leq 0.5,\end{cases}
$$




$$
\lambda_{k}(\mu)=\left\{\begin{array}{l}
0, \text { if } \mathrm{k}=0 \\
\mu, \text { if } \mathrm{k} \in\{1,2, \ldots,\}
\end{array}\right.
$$

The hybrid fuzzy IVP (4.4) is equivalent to the following systems of fuzzy IVPs.

$$
\left\{\begin{array}{l}
y_{0}{ }^{\prime}(t)=y_{0}(t), t \in[0,1] \\
y_{0}(0 ; r)=[(0.75+0.25 r) e,(1.125-0.125 r) e], 0 \leq \mathrm{r} \leq 1 \\
y_{i}^{\prime}(t)=y_{i}(t)+m(t) y_{i-1}(t), \mathrm{t} \in\left[t_{i}, t_{i+1}\right], y_{i}(t)=y_{i-1}\left(t_{i}\right), i=1,2, \ldots,
\end{array}\right.
$$

In (4.4) $y(t)+m(t) \lambda_{k}\left(y\left(t_{k}\right)\right)$ is continuous function of $\mathrm{t}, \mathrm{x}$ and $\lambda_{k}\left(y\left(t_{k}\right)\right)$. For each $\mathrm{k}=0,1,2, \ldots$, the fuzzy IVP

$$
\left\{\begin{array}{l}
y^{\prime}(t)=y(t)+m(t) \lambda_{k}\left(y\left(t_{k}\right)\right), t \in\left[t_{k}, t_{k+1}\right], t_{k}=k \\
y\left(t_{k}\right)=y_{t_{k}},
\end{array}\right.
$$

has a unique solution on $\left[t_{k}, t_{k+1}\right]$. To numerically solve the hybrid fuzzy IVP (4.4)we will apply the fourth order Runge - Kutta method based on Geometric Mean for hybrid fuzzy differential equation with $\mathrm{N}=2$ to obtain $\mathrm{y}_{1,2}(\mathrm{r})$ approximating $\mathrm{Y}(2.0 ; \mathrm{r})$. Let $f:[0, \infty) \times R \times R \rightarrow R$ be given by

$$
f\left(t, y, \lambda_{k}\left(y\left(t_{k}\right)\right)\right)=y(t)+m(t) \lambda_{k}\left(y\left(t_{k}\right)\right), t_{k}=k, k=0,1,2, \ldots
$$

where $\lambda_{k}: R \rightarrow R$ is given by

$$
\lambda_{k}(y)=\left\{\begin{array}{l}
0, \text { if } \mathrm{k}=0 \\
y, \text { if } \mathrm{k} \in\{1,2, \ldots,\}
\end{array}\right.
$$

Since the exact solution of (4.4) for $\mathrm{t} \in[1,1.5]$ is

$$
Y(t ; r)=Y(1 ; r)\left(3 e^{t-1}-2 t\right), 0 \leq r \leq 1, \mathrm{Y}(1.5 ; \mathrm{r})=\mathrm{Y}(1 ; \mathrm{r})(3 \sqrt{e}-3), 0 \leq \mathrm{r} \leq 1 \text {. }
$$

Then $\mathrm{Y}(1.5 ; \mathrm{r})$ is approximately 5.29 and $\mathrm{y}_{1,1}$ is approximately 5.255963 . Since the exact solution for (4.4) for $\mathrm{t}[1.5,2]$ is

$$
Y(t ; r)=Y(1 ; r)\left(2 t-2+e^{t-1.5}(3 \sqrt{e}-4)\right), 0 \leq r \leq 1
$$

Therefore

$$
\mathrm{Y}(2.0 ; \mathrm{r})=\mathrm{Y}(1 ; \mathrm{r})(2+3 \mathrm{e}-4 \sqrt{e}) .
$$

Then $\mathrm{Y}(2.0 ; \mathrm{r})$ is approximately 9.6769 and $\mathrm{y}_{11}(2.0 ; 1)$ is approximately 9.623694 . The absolute errors by RK4GM and the classical fourth order RK method for the $\mathrm{r}$-level set with $\mathrm{h}=0.5$ and for $\mathrm{t}=1.5,2$ of example 4.1 is given in Table 4.1. The graphical representation for the approximate values between RK4GM and the classical fourth order RK method of example 4.1 is given in figure.4.1.

Table 4.1.

The absolute error table for example 4.1 for $\mathrm{r}$ - level set with $\mathrm{h}=0.5$ and $\mathrm{t}=1.5,2$.

\begin{tabular}{|c|c|c|c|c|c|c|c|c|}
\hline $\mathrm{r}$ & \multicolumn{3}{|c|}{ Absolute Error Value $(\mathrm{h}=0.5$ and $\mathrm{t}=1.5)$} & \multicolumn{2}{c|}{ Absolute Error Value $(\mathrm{h}=0.5$ and $\mathrm{t}=2)$} \\
\hline & \multicolumn{2}{|c|}{ HFRK4GM } & \multicolumn{2}{c|}{ HFRK4AM } & \multicolumn{2}{c|}{ HFRK4GM } & \multicolumn{2}{c|}{ HFRK4AM } \\
\hline 0 & $2.57 \mathrm{E}-02$ & $3.85 \mathrm{E}-02$ & $2.89 \mathrm{E}-03$ & $4.34 \mathrm{E}-03$ & $4.00 \mathrm{E}-02$ & $5.99 \mathrm{E}-02$ & $5.32 \mathrm{E}-03$ & $7.97 \mathrm{E}-03$ \\
\hline 0.1 & $2.66 \mathrm{E}-02$ & $3.81 \mathrm{E}-02$ & $2.99 \mathrm{E}-03$ & $4.29 \mathrm{E}-03$ & $4.13 \mathrm{E}-02$ & $5.93 \mathrm{E}-02$ & $5.49 \mathrm{E}-03$ & $7.88 \mathrm{E}-03$ \\
\hline 0.2 & $2.74 \mathrm{E}-02$ & $3.77 \mathrm{E}-02$ & $3.09 \mathrm{E}-03$ & $4.24 \mathrm{E}-03$ & $4.26 \mathrm{E}-02$ & $5.86 \mathrm{E}-02$ & $5.67 \mathrm{E}-03$ & $7.80 \mathrm{E}-03$ \\
\hline 0.3 & $2.83 \mathrm{E}-02$ & $3.73 \mathrm{E}-02$ & $3.18 \mathrm{E}-03$ & $4.19 \mathrm{E}-03$ & $4.40 \mathrm{E}-02$ & $5.79 \mathrm{E}-02$ & $5.85 \mathrm{E}-03$ & $7.71 \mathrm{E}-03$ \\
\hline 0.4 & $2.91 \mathrm{E}-02$ & $3.68 \mathrm{E}-02$ & $3.28 \mathrm{E}-03$ & $4.15 \mathrm{E}-03$ & $4.53 \mathrm{E}-02$ & $5.73 \mathrm{E}-02$ & $6.02 \mathrm{E}-03$ & $7.62 \mathrm{E}-03$ \\
\hline 0.5 & $3.00 \mathrm{E}-02$ & $3.64 \mathrm{E}-02$ & $3.37 \mathrm{E}-03$ & $4.10 \mathrm{E}-03$ & $4.66 \mathrm{E}-02$ & $5.66 \mathrm{E}-02$ & $6.20 \mathrm{E}-03$ & $7.53 \mathrm{E}-03$ \\
\hline 0.6 & $3.08 \mathrm{E}-02$ & $3.60 \mathrm{E}-02$ & $3.47 \mathrm{E}-03$ & $4.05 \mathrm{E}-03$ & $4.80 \mathrm{E}-02$ & $5.59 \mathrm{E}-02$ & $6.38 \mathrm{E}-03$ & $7.44 \mathrm{E}-03$ \\
\hline 0.7 & $3.17 \mathrm{E}-02$ & $3.55 \mathrm{E}-02$ & $3.57 \mathrm{E}-03$ & $4.00 \mathrm{E}-03$ & $4.93 \mathrm{E}-02$ & $5.53 \mathrm{E}-02$ & $6.56 \mathrm{E}-03$ & $7.35 \mathrm{E}-03$ \\
\hline 0.8 & $3.25 \mathrm{E}-02$ & $3.51 \mathrm{E}-02$ & $3.66 \mathrm{E}-03$ & $3.95 \mathrm{E}-03$ & $5.06 \mathrm{E}-02$ & $5.46 \mathrm{E}-02$ & $6.73 \mathrm{E}-03$ & $7.26 \mathrm{E}-03$ \\
\hline 0.9 & $3.34 \mathrm{E}-02$ & $3.47 \mathrm{E}-02$ & $3.76 \mathrm{E}-03$ & $3.90 \mathrm{E}-03$ & $5.20 \mathrm{E}-02$ & $5.39 \mathrm{E}-02$ & $6.91 \mathrm{E}-03$ & $7.18 \mathrm{E}-03$ \\
\hline 1 & $3.43 \mathrm{E}-02$ & $3.43 \mathrm{E}-02$ & $3.86 \mathrm{E}-03$ & $3.86 \mathrm{E}-03$ & $5.33 \mathrm{E}-02$ & $5.33 \mathrm{E}-02$ & $7.09 \mathrm{E}-03$ & $7.09 \mathrm{E}-03$ \\
\hline
\end{tabular}




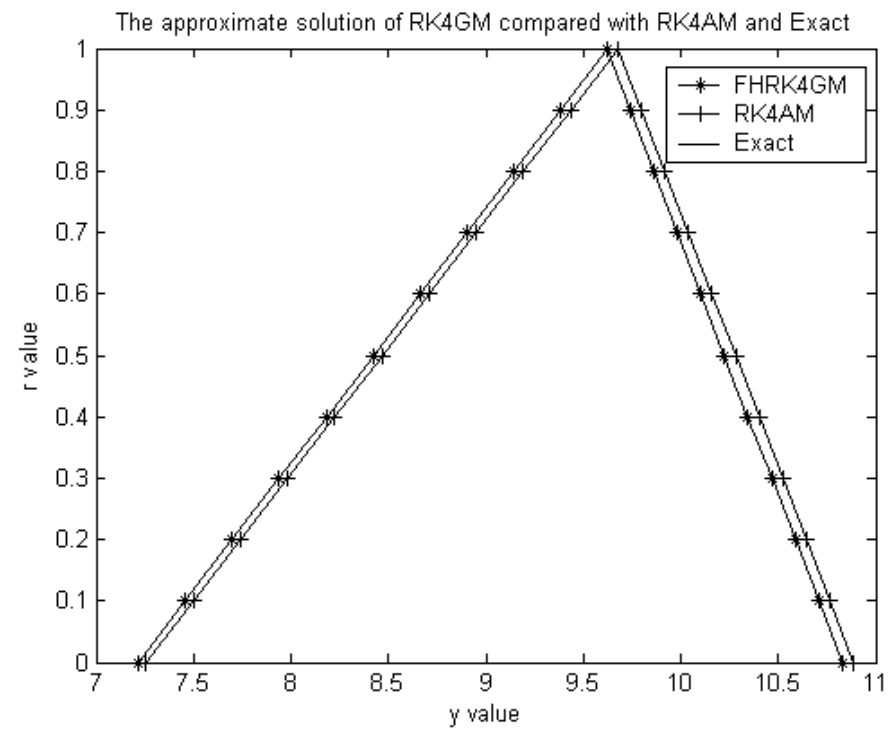

Figure 4.1 Comparison of RK4GM with RK4AM and the Exact Solution when $\mathrm{N}=2$ and $\mathrm{t}=2$

\section{Conclusion}

In this work, the proposed fourth order Runge-Kutta method based on Geometric Mean for finding the numerical solution of hybrid fuzzy initial value problem works very well. From the Tables 4.1 figure 4.1 of example 4.1, it is shown that the fourth order Runge- Kutta method based on Geometric Mean is suitable for solving hybrid fuzzy initial value problem.

\section{Acknowledgements}

This work has been supported by University Grants Commission, MRP - 5841/15, SERO, Hyderabad, India.

\section{References}

[1]. Abbasbandy. S, Allah Viranloo. T, Numerical solution of fuzzy differential equations by Runge-Kutta method, Nonlinear studies.11(2004)N0.1,117-129.

[2]. Abbasbandy. S, Allahviranloo. T, Numerical solution of fuzzy differential equations by Taylor method, Journal of Computational Methods and Applied Mathematics, 2(2002)113-124.

[3]. Abdul-Majid Wazwaz, A comparision of modified Runge-Kutta formulas based on a variety of means, International Journal of Computer Mathematics, vol.50,pp-105-112.

[4]. Balachandran. K, Prakash. P, Existence of solutions of fuzzy delay differential equations with nonlocal condition, Journal of the Korea Society for Industrial and Applied Mathematics, 6(2002)81-89.

[5]. Balachandran. K, Kanagarajan. K, Existence of solutions of fuzzy delay integrodifferential equations with nonlocal condition, Journal of the Korea Society for Industrial and Applied Mathematics, 9(2005)65-74.

[6]. Chang. S.L, Zadeh. L.A, On fuzzy mapping and control, IEEE Trans, Systems Man Cybernet. 2(1972)30-34.

[7]. Dubois. D, Prade. H, Towards fuzzy differential calculus part 3 : Differentiation, Fuzzy Sets and Systems, 8(1982)225-233.

[8]. Evans. D.J \& Sanugi. B.B. (1991). A comparison of numerical o.d.e. solvers based on arithmetic and geometric means. International Journal of Computer Mathematics, 32 - 35.

[9]. Jayakumar. T and Kanagarajan. K, "Numerical solution for hybrid fuzzy system by Runge-Kutta method of order five", Applied Mathematical Sciences, Vol. 6,2012, no.72, 3591 - 3606

[10]. Kaleva. O, Fuzzy differential equations, Fuzzy Sets and Systems, 24(1987)301-317.

[11]. Kaleva. O, The Cauchy problem for fuzzy differential equations, Fuzzy Sets and Systems, 35(1990)389 -396.

[12]. Kanagarajan. K, Sambath. M , Runge- Kutta Nystrom method of order three for solving fuzzy differential equations, Computational methods in Applied Mathematics, Vol.10(2010), No.2, pp.195-203.

[13]. Kanagarajan. K, Sambath. M ,Numerical solution of fuzzy differential equations by third order Runge-Kutta method, International journal of Applied Mathematics and Computaion , 2(4) (2010) pp.1-8.

[14]. Lambert. J.D, Numerical Methods for ordinary differential systems, John Wiley \&Sons, 1990.

[15]. Ma. M, Friedman. M, Kandel. A, Numerical solutions of fuzzy differential equations, Fuzzy Sets and Systems, 105(1999)133-138.

[16]. Murugesan. K, Paul Dhayabaran. D, Henry Amirtharaj. E.C and David J. Evans , Numerical Strategy for the system of second order IVPs using RK method based on Centroidal Mean.Intern.J.Comp.Math.80(2), (2003), pp.233-241.

[17]. Murugesan. K, Paul Dhayabaran. D, Henry Amirtharaj. E.C and David J. Evans , A Comparison of extended Runge - Kutta formulae based on Variety of means to solve system of IVPs, Intern.J.Comp.Math.78 (2001), pp.225-252.

[18]. Murugesan. K, Paul Dhayabaran. D, Henry Amirtharaj. E.C and David J. Evans , A fourth order embedded Runge - Kutta RKACeM $(4,4)$ method based on Arithmetic and Centroidal means with error conrol, Intern.J.Comp.Math.79(2) (2002), pp.247-269.

[19]. Nirmala. V, Chenthur Pandian. S, "Numerical Solution of Fuzzy Differential Equation by Fourth Order Runge-Kutta Method with Higher Order Derivative Approximations", European Journal of Scientific Research, Vol.62 N0.2 (2011),pp.198-206.

[20]. Palligkinis. S.ch., Papageorgiou. G, Famelis,I.TH., "Runge-Kutta methods for fuzzy differential equations", App.Math.Comp 209(2009),97-105. 
Fourth Order Runge-Kutta Method Based On Geometric Mean for Hybrid Fuzzy Initial Value ..

[21]. Pederson.S and Sambandham, "Numerical solution to hybrid fuzzy systems",Mathematical and Computing Modelling, vol.45, no. 910,pp.1133-1144, 2007.

[22]. Pederson.S and Sambandham.M, "The Runge-Kutta method for hybrid fuzzy differential equations", Nonlinear Analysis: hybrid systems 2,pp.626-634,2006.

[23]. Prakash.P and Kalaiselvi. V, "Numerical solution of hybrid fuzzy differential equations by predictor-corrector method", International Journal of Computer Mathematics, vol.86, no.1.pp.121-134, 2009.

[24]. Puri. M.L, Ralescu. D.A, Differentials of fuzzy functions, Journal of Mathematical Analysis and Applications, 91(1983)552-558.

[25]. Saveetha.N and Chendur Pandian.S,"Numerical solution of fuzzy hybrid differential equation by third order nystrom method", Mathematical Theory and Modeling, vol.2,No.4,2012

[26]. Seikkala. S, On the fuzzy initial value problem, Fuzzy Sets and Systems, 24(1987)319-330. 